\title{
SPERMATOZOA: THE PROBABLE SELECTION OF A SMALL POPULATION BY THE GENITAL TRACT OF THE FEMALE RABBIT
}

\author{
J. COHEN ANd DENISE C. MaNAUGHTON \\ Department of Zoology and Comparative Physiology, University of Birmingham, \\ P.O. Box 363, Birmingham B15 2TT
}

(Received 16th August 1973)

\begin{abstract}
Summary. Spermatozoa recovered from rabbit uterus or oviducts, or from antibody columns, were mixed with freshly ejaculated spermatozoa of different genotype and inseminated into the uterus of a second doe, 9 to $12 \mathrm{hr}$ before ovulation to allow time for capacitation. Forty-seven litters were produced. Spermatozoa from high in the female tract were individually more potent: $1.9 \times 10^{7}$ 'unselected', $2.1 \times 10^{7}$ 'chemically selected', $7 \times 10^{5}$ uterine, $1.6 \times 10^{4}$ oviducal spermatozoa were inseminated per offspring produced. If only spermatozoa represented by progeny were counted, the figures became: $1.6 \times 10^{7}, 3.4 \times 10^{6}, 2.7 \times 10^{5}$, $1.04 \times 10^{2}$, respectively. Logarithmic means, for litters calculated separately, were: unselected $6 \cdot 61 \pm 0 \cdot 19$, chemically selected $5 \cdot 92 \pm 0 \cdot 42$, uterine selected $4 \cdot 91 \pm 0 \cdot 45$, oviducally selected $2 \cdot 04 \pm 0 \cdot 17$.

This is considered good evidence that part of the function of the mammalian female tract is selection of a few spermatozoa for fertilization. Possible reasons for such selection are considered, and the process is related to errors of chiasmata during spermatogenesis.
\end{abstract}

\section{INTRODUCTION}

Diverse explanations have been offered for the enormous numbers of spermatozoa ejaculated by many species of animals (review by Cohen, 1971).

Parker (1970), among others, has suggested that males which produce large numbers of spermatozoa are at a selective advantage when several males mate with each female, and therefore that sperm numbers will rise in the course of evolution. Some workers have proposed the view that the huge numbers overwhelm defences designed primarily to protect the open female tract against bacterial invasion (e.g. Cohen, Jean, 1969). Others (e.g. Restall, 1967) suggest that spermatozoa might lose their way in the circuitous female genital tracts. All these explanations use 'force of numbers' in different ways, but all imply that any individual spermatozoon which arrives at the egg can fertilize it.

A second class of explanation involves timing. Delays are apparently necessary in mammals, for sperm capacitation and often for maturation of the ovum. Austin (1965) has proposed that the various barriers in the female tract result 
in a continuous trickle of a few spermatozoa to the fertilization site. The enormous reservoir ensures that the trickle persists until the eggs are available. This avoids too many spermatozoa around the egg, which might cause polyspermy or even digestion of the whole vitellus (e.g. Adams, 1969). This theory also implies that any ripe spermatozoon that meets the mature egg can fertilize.

The third class of explanation supposes that spermatozoa differ, only a very small proportion being acceptable gametes and these being the few which are allowed by the female tract to reach the fertilization site (e.g. Cohen, 1967). Spermatozoa produced with defects introduced during spermatogenesis remain in the ejaculate because selection is left to the female. The total number ejaculated would necessarily be at least the reciprocal of the fertile proportion, e.g. if only a thousandth are acceptable gametes, then more than a thousand must be offered. This class of explanation sees the surviving spermatozoa as an acceptable minority, the defective majority having been separated from them in the female tract.

What occurs in the female tract of the mammal varies greatly between species, but all restrict sperm numbers at the fertilization site.

In the rabbit, ovulation follows 9 to $13 \mathrm{hr}$ after mating (Harper, 1963; Adams, 1972). About $10 \mathrm{hr}$ is needed for optimum sperm capacitation (Soupart, 1967; Bedford, 1970). The number of spermatozoa deposited in the cervical end of the vagina is about $6 \times 10^{7}$ to $2.0 \times 10^{8}$; Mann (1964) gives a mean of $1.5 \times 10^{8}$. The number negotiating the cervix is apparently small, as the number in the uterus rises slowly to a maximum of some $4 \times 10^{6}$ about 8 to $10 \mathrm{hr}$ after coitus; the numbers in the oviducts are much smaller, a few thousands (Braden \& Austin, 1954; Morton, 1970). At ovulation, some hundreds of spermatozoa at most are in the oviducal ampulla, and it must be some of these which fertilize.

About $4 \mathrm{hr}$ after coitus, there is a massive invasion of the uterine lumen by leucocytes. A small number, probably about $1 \times 10^{6}$, may be found in the uterus before coitus, but the new arrivals number $1.0 \times 10^{7}$ or more. A large proportion of the spermatozoa are subsequently found attached to macrophages and many are engulfed (e.g. Bedford, 1965, 1970). This phagocytosis is associated with antibody coating of the spermatozoa (Symons, 1967; Edwards, 1967; Bedford, 1970). Nevertheless, the number of spermatozoa in the uterus remains steady, or rises slightly until about 10 to $12 \mathrm{hr}$ after coitus, when there is a fall, and by $15 \mathrm{hr}$ ( $2 \mathrm{hr}$, or so, after ovulation) only $0.5 \times 10^{6}$ at most can be found. A few motile spermatozoa seem to persist, even up to $90 \mathrm{hr}$ after coitus (Morton, 1970), so selection cannot be proved or excluded by these observations.

We decided to test whether the surviving population of spermatozoa in the rabbit was a special proportion which had been selected, or overspill from large numbers inseminated into the female tract. In the first case, samples recovered progressively nearer to fertilization in place and time should decline in numbers but show less decline in fertility: fewer spermatozoa should do better, and those from the oviducts should do best. Alternatively, surviving spermatozoa would not be expected to fertilize as readily as fresh (but capacitated) spermatozoa. To compare recovered spermatozoa with fresh spermatozoa 
by direct competition in mixed inseminates, and to counter ambiguity resulting from different capacitation of the selected spermatozoa, the recipient female was injected with HCG or mock-mated at or near the time of insemination. In this way, capacitation time would be optimal for the competing unselected spermatozoa and the 'test' spermatozoa would reside in two female tracts for considerably longer than the 10- to 12-hr optimum. To distinguish technical failures (null results) from inadequacy of the inseminated spermatozoa (negative results), adequate numbers of unselected spermatozoa, more than were found necessary by Wales, Martin \& O'Shea (1965) but less than were introduced during a normal intromission, were always used.

Although many workers (e.g. Edwards, Bavister \& Steptoe, 1969) have used penetration or cleavage as an index of fertilization, our use of genetic markers necessarily involved examination of the full-term progeny.

\section{MATERIALS AND METHODS}

Thirty-seven buck rabbits were obtained from various suppliers and breeders, as pure chinchillas or agouti lops, and all were tested for pigment genetics before use: when test mated with New Zealand White (NZW) does, three small-eared chinchillas and three agouti lops produced offspring resembling or clearly assignable to the father, both in pigmentation and ear character, and they all continued to do so in test mating during the experimental series. Bucks from the same NZW strains as the does were also used.

The bucks were allowed to mate, or semen was collected using an artificial vagina (Holborn Surgical Co., London), between one and four times weekly; semen quality was monitored for sperm number and motility. Two NZW bucks were vasectomized; the sperm count was zero after 9 weeks and was periodically checked. Libido seemed unaffected. All does used were of NZW commercial high-production strains (but naturally had a great variety of colour genes) and were obtained from Hylyne Rabbits until June 1972 and from Cheshire Rabbit Farms latterly. All does were supplied as 'New Zealand White doe which has had one litter', and weighed 3.5 to $4.5 \mathrm{~kg}$.

All animals were kept in individual cages with hay and sawdust, and had free access to 'TR2 + extra vit G' (Pilsbury) rabbit diet, supplemented twice weekly with $250 \mathrm{~g}$ fresh cabbage and a carrot per animal. Fresh water was supplied daily. The rabbits were at first kept under natural light conditions, but this was changed to $14 \mathrm{hr}$ light in February 1972. In March 1972, the room temperature was reduced from $24^{\circ} \mathrm{C}$ to below $20^{\circ} \mathrm{C}$ as far as this was possible during summer months, and to approximately $16^{\circ} \mathrm{G}$ in winter. The change of supplier was made after a long period of poor littering, and the situation improved.

Ejaculates were collected into a Bijou bottle warmed to prevent cold-shock to the spermatozoa. They were routinely suspended in $10 \mathrm{ml}$ Tissue Culture Medium 199 with Hanks salts, bicarbonate or HEPES buffer (Bio-Cult or Flow Labs) and 1 i.u. penicillin $\mathrm{G}$ and 1 i.u. streptomycin sulphate $/ \mathrm{ml}$, and were allowed to settle in a $37^{\circ} \mathrm{C}$ water-bath for 5 to $10 \mathrm{~min}$. They were then decanted into another tube, centrifuged at $1600 \mathrm{~g}$ for $10 \mathrm{~min}$, and resuspended 
in a small volume (about $0.5 \mathrm{ml}$ ) of Medium 199. Flow Labs round-bottomed 15-ml screw-top tissue culture tubes were used.

When a vasectomized buck was not available (all experiments till December 1971, and sporadically thereafter), 50 to 75 i.u. HCG (Lutormone, Burroughs Wellcome) was injected into the ear vein to induce ovulation in the recipient doe, often following the Nembutal anaesthetic.

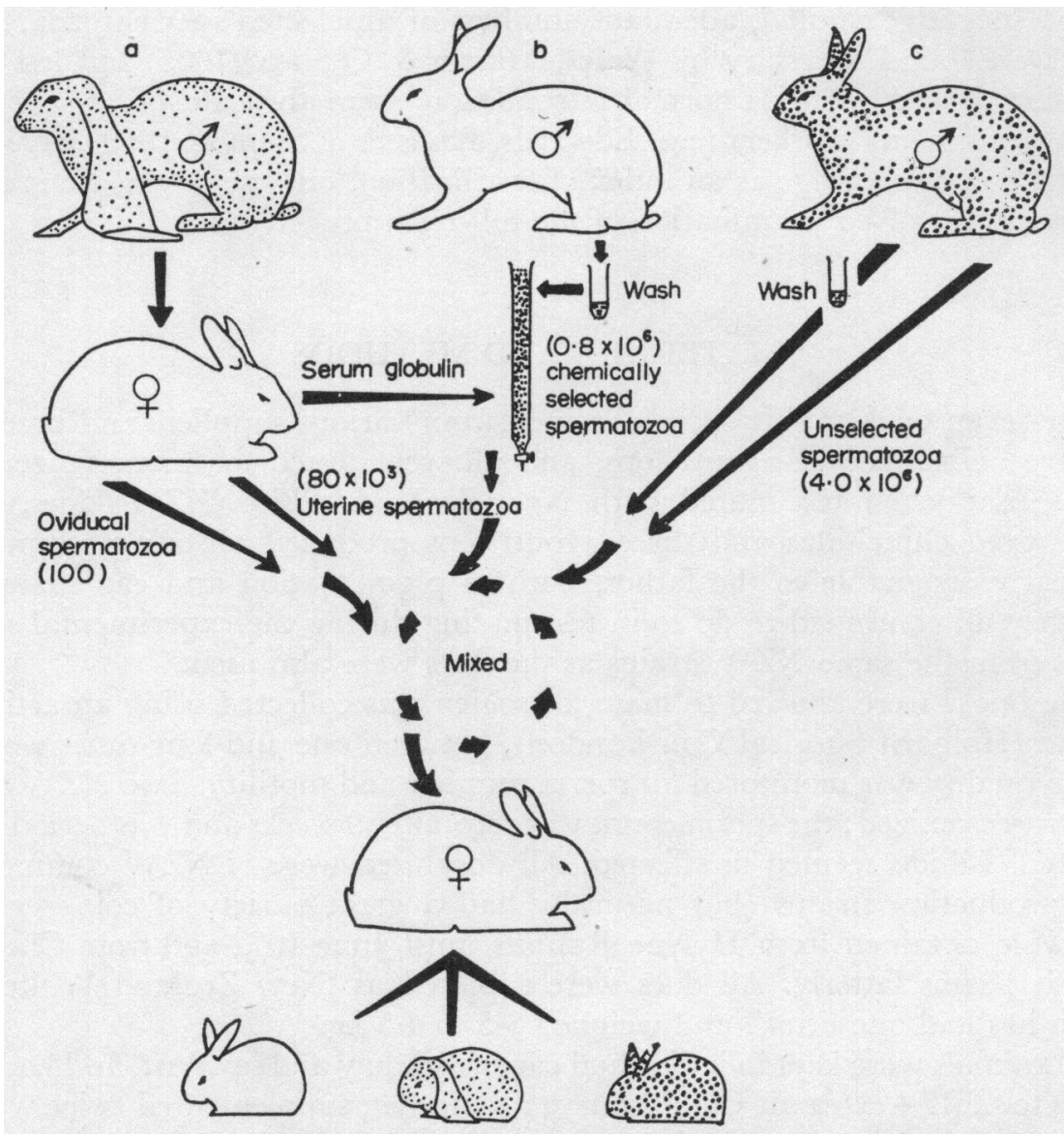

Text-Fig. 1. The experimental design. Different breeds (agouti lop, albino and chinchilla) were used as a, b and $c$ in successive experiments. The numerical values, see Table 2, are numbers of spermatozoa/offspring for each route. Usually only two sources of spermatozoa were mixed and inseminated.

A method was developed to separate spermatozoa which did not stick to globulin. Blood was drawn from the heart of filter does, and the serum was kept at $4^{\circ} \mathrm{C}$ overnight; then the globulin fraction was precipitated using chilled saturated ammonium sulphate of equal volume. This was centrifuged at $9500 \mathrm{~g}$ for $20 \mathrm{~min}$, dialysed against glass-distilled water and stirred at $4^{\circ} \mathrm{C}$ until $2 \%$ barium chloride gave no turbidity with the dialysate (about twenty 2-litre changes in $6 \mathrm{hr}$ ). Globulin solution was then stored frozen at $-20^{\circ} \mathrm{C}$. Glass 
bead columns ( 40 mesh) were washed through with $5 \%$ horse or rabbit serum and then baked at $120^{\circ} \mathrm{C}$ for 60 to $90 \mathrm{~min}$. After cooling, they were filled with acetone and left full for more than $10 \mathrm{~min}$. After one short rinse with Oxoid Dulbecco A phosphate-buffered saline (PBS), the globulin solution was added and allowed to remain in contact with the acetone-soaked protein matrix until semen was ready ( 1 to $3 \mathrm{hr}$ ). After three brief or two longer rinses with PBS, semen or washed spermatozoa were allowed to percolate through the beads, followed by Medium 199. The spermatozoa that were not retained were collected in about $2 \mathrm{ml}$, scored for motility, agglutination state and approximate number and then kept ready for use in the $37^{\circ} \mathrm{C}$ water bath. In some experiments, spermatozoa collected from the columns were subjected to immunofluorescent staining to determine their antibody/antigen-coating status. This work is being prepared for publication elsewhere.

The experimental design is shown in Text-fig. 1. With minor variations, each experiment was conducted as follows: at 07.00 hours, a doe (the filter) was mated with one buck, so that spermatozoa could later be found in the upper tract. A buck of a different breed would fill this rôle the following week. After 11.00 hours, a second doe (the recipient) was 'mated' with the vasectomized buck, or injected with HCG (usually at 14.00 hours), to ensure ovulation about midnight or later. At about 14.00 hours, the recipient doe was anaesthetized with an injection of 2.5 to $4 \mathrm{ml} \mathrm{Nembutal}$ (Abbott) into an ear vein, the abdomen was shaved over the umbilicus, and a 2- to 3-cm laparotomy incision was made to expose and examine the uterine horns. The wound was temporarily clipped with four 12-mm Michele clips and the doe, wrapped in a towel to help maintain her temperature, was removed from the electrically warmed operating table; this procedure enabled uterine insemination to be carried out with minimal delay.

Semen samples were now collected from bucks of the two breeds which had not been used at first. One sample was washed in Medium 199 as described above, the other was allowed to percolate slowly down the glass-bead column.

The filter doe was then (about 15.25 to 15.35 hours) overdosed with 5 to $10 \mathrm{ml} \mathrm{Nembutal}$ in the ear vein and exsanguinated from the heart so that there would be less likelihood of blood contamination of the sperm samples and to provide globulin for the following week's experiment. Throughout, body temperature was not allowed to fall as this provoked uterine contractions and the recovery of large, presumably spurious, numbers of spermatozoa from the tubes. The abdomen was opened, and the uterus and tubes were exposed and clamped with artery forceps at the uterovaginal and both uterotubal junctions, usually within 1 min of cessation of heart-beat. Each oviduct was dissected free of associated connective tissue after filling to distension through the funnel with warm HEPES-buffered Medium 199 (usually $1 \mathrm{ml}$ ) and clamping with an artery forceps. Fat was carefully removed, with a cotton bud, from the uterine end of a 2-cm segment of the tube, and this was cut and allowed to drain into a 4-ml glass receiver; air was then blown through the tube to flush it, and usually the same medium was flushed through again. The same medium was usually also used for the other tube. Both uterine horns were then flushed, using about $2 \mathrm{ml}$ Medium 199 and $2 \mathrm{ml}$ air to assist recovery of the liquid. 
One drop of the tubai flushings was examined by phase contrast 150, scan ning the complete 12-mm coverslip; if more than about ten spermatozoa were found per field, or any leucocytes or erythrocytes, the sample was discarded (and spermatozoa recovered from uterus or column were later inseminated instead). If no spermatozoa were found (about two in three of the early experif ments and one in three of the later ones), the sample was added to the uterine flushings. The uterine flushings were also examined microscopically, and sperm number, motility and agglutination state as well as number of leucocytes was assessed. Samples ofboth flushings, and of unselected spermatozoa and chemic 7 ally selected' spermatozoa were taken for counting (see below). Two sperm suspensions of known volumes were then mixed and inseminated into both horns of the uterus of the recipient doe. The wound was stitched in two layers, dusted with sulphanilamide powder, and sprayed with Nobecutane (BDH). Recovery from the anaesthetic was complete by 18.00 hours, and little trouble was experienced from wound infections; two does were killed because they showed persistent poor wound closure, but nearly all the does showed evidence of clean healing without adhesions when they were mated and killed to assess such damage; usually they were used as filters. The recipient does were trans ferred to larger breeding cages some days before kindling was expected, and given extra hay and greens.

Samples $(0-02 \mathrm{ml})$ from the unselected, 'chemically selected' and uterine sperm suspensions were diluted according to different protocols, and one $0-02-\mathrm{ml}$ drop of each final dilution was dried on each of three chemically clean slides. The oviducal flushings each provided three 0-02-ml drops directly, without dilution. These were then stained in Casaret's stain (Casaret, 1953), rinsed, air-dried, and permanently mounted in DPX. Spermatozoa were counted only if the female littered, and after the paternity had been assigned; the original sperm numbers inseminated were calculated from counts of the three slides, the volume inseminated and the dilution protocol. Cohen (1971) justified this counting procedure and gives more detail.

\section{RESULTS}

The paternity of the progeny of the NZW does was always easily assignable to the three buck breeds. The NZW bucks always produced progeny resembling the parents in all respects : albino, with erect ears. The agouti lops produced, with the varying doe genetics, a variety of colour : agouti, black, piebald, fawn, and

\section{EXPLANATION OF PLATE 1}

Some of the parents and offspring rabbits used and obtained in the experiments reported. FIG. 1. The mother, and offspring at 4 weeks, of the first successful 'oviducal' experiment. The two bucks are shown as insets.

FIG. 2. The same litter at 6 weeks. The ears of the brown and black rabbits have lopped fully, those of the white not at all. Inset is a control white offspring from a heterozygousaibino lop buck and NZW doe at 5 weeks (see text).

FIG. 3. Some chinchilla 'oviducal' progeny with their parents (chinchilla buck and offspring, left; NZW doe and offspring, right), and the NZW buck (centre) which provided unsuccessful unselected spermatozoa. 
PLATE 1
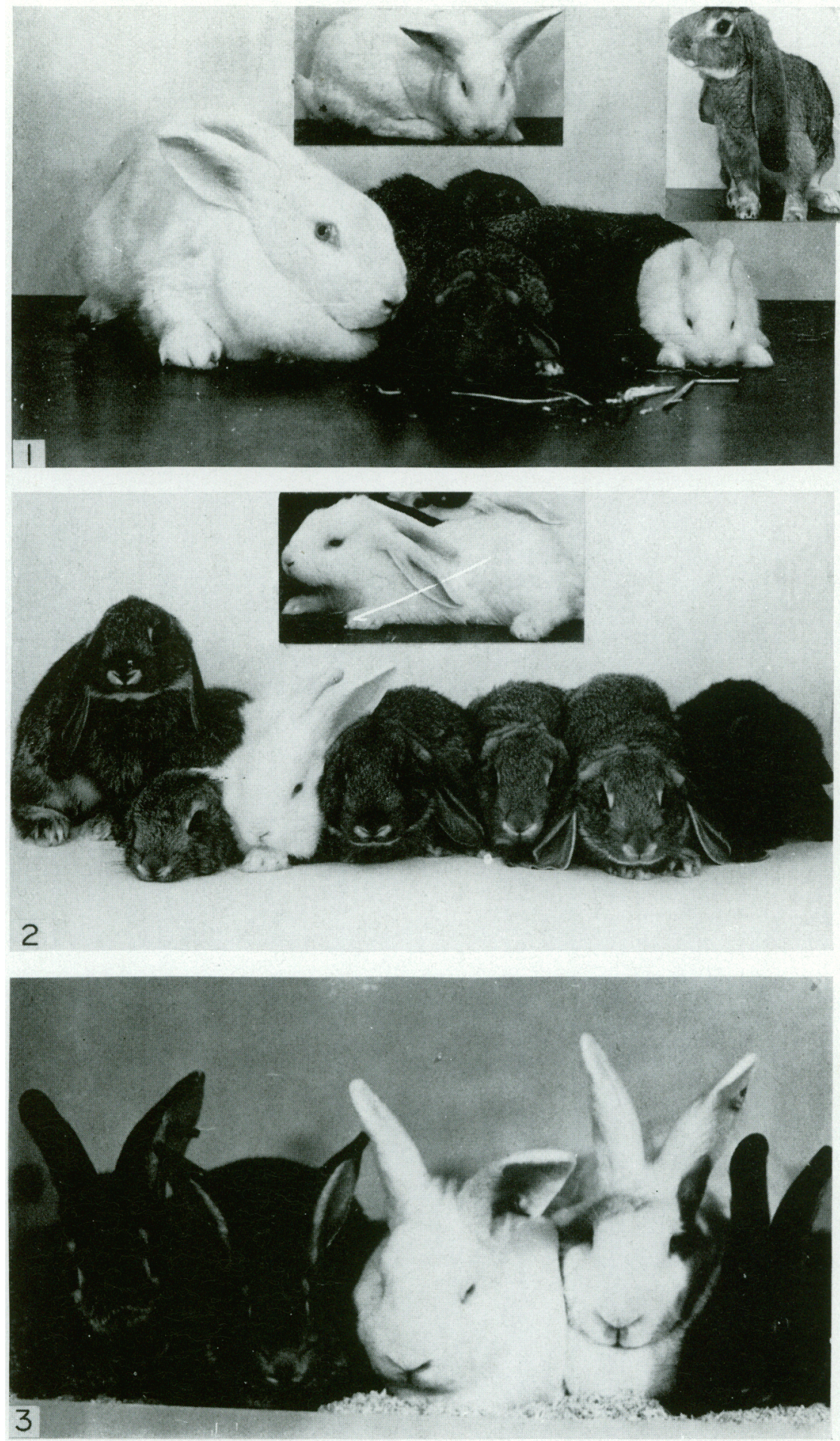

(Facing p. 302) 
sooty fawn, but always with obvious large lop ears at 6 weeks (Plate 1). The chinchilla buck produced a variety: chinchilla, 'ghost' chinchilla, black fox-pattern, Himalayan with pigmented eyes, or spotted ('English'), but always with short ears. Where colour pattern could have come from either agouti lop or chinchilla father, ear length was always very different, but, in the event, none of the experimental litters produced this problem. Usually, a pigmented and a NZW buck were in question.

Our bucks produced between $5 \times 10^{6}$ and $5 \times 10^{8}$ spermatozoa per ejaculate; this was not apparently related to breed or volume, except that two of the three lop bucks tended to produce high volumes with low concentration and motility. After washing, $1 \cdot 8 \pm_{0.5}^{0.6} \times 10^{7}$ were used as the unselected component of the mixed inseminates $(\log$ means \pm S.E. $=7 \cdot 24 \pm 0 \cdot 13)$ because this number had been shown to be adequate for full fertility in a pilot series. [The sperm numbers form a normal distribution on a logarithmic scale and the log mean numbers of spermatozoa \pm S.E. (e.g. $7 \cdot 24 \pm 0 \cdot 13$ ) have been expressed in this way. For convenience, the arithmetic means (e.g. $1 \cdot 8 \times 10^{7}$ ) are also given and the asymmetry of the variance has been expressed in arithmetic terms (e.g. $\left.\left.\pm_{0.5}^{0.6}\right) \cdot\right]$

The number of spermatozoa found in the uterine washings of the filter doe 6 to $8 \mathrm{hr}$ after copulation was $9 \cdot 8 \pm \pm_{4 \cdot 4}^{10 \cdot 2} \times 10^{4}(4 \cdot 99 \pm 0 \cdot 28)$. These were usually strongly motile but in most cases were accompanied by a great excess of leucocytes, to which a variable proportion of the spermatozoa were attached.

The number of spermatozoa found in the oviducal washings of the filter doe 6 to $8 \mathrm{hr}$ after copulation was $1 \cdot 1 \pm_{0 \cdot 4}^{0 \cdot 6} \times 10^{3}(3 \cdot 02 \pm 0 \cdot 22)$.

The number of spermatozoa which emerged from the column, not having stuck to the globulin, was $4 \cdot 3 \pm 2 \cdot 1.9 \times 10^{6}(6 \cdot 63 \pm 0 \cdot 28)$. Their condition was also very variable, i.e. strongly agglutinated, free, strongly motile or barely twitching.

The recipient does produced progeny after forty-seven of the 200 operations performed; the others were considered technical failures (null experiments) because excess, or at least adequate, numbers of unselected spermatozoa had been inseminated in all cases. Eighteen of these litters contained progeny from selected spermatozoa; only four litters contained progeny from spermatozoa flushed from the oviducts. A total of $3.8 \times 10^{9}$ unselected spermatozoa were inseminated and produced 198 young; $4.4 \times 10^{8}$ 'chemically selected' spermatozoa produced twenty-one young; $2.2 \times 10^{7}$ uterine spermatozoa produced thirty-one young; $1.4 \times 10^{5}$ oviducal spermatozoa produced nine young. Tables 1 and 2 present all the data (more than 200 experiments were performed as test matings formed part of the series).

There was little or no difference between the breeds in the number of spermatozoa required/offspring, although the figures are not really adequate to justify this conclusion. The $\log$ means \pm S.E. for the buck breeds are: agouti lop, $6 \cdot 58 \pm 0 \cdot 3 ; \mathrm{NZW}, 6 \cdot 87 \pm 0 \cdot 27$; chinchilla, $6 \cdot 01 \pm 0 \cdot 35$.

Eighteen young were recovered stillborn or from the uteri of two does which were killed. Since, in these cases, spermatozoa came from an albino (NZW) and a pigmented father, examination of the eyes of the offspring (six pigmented, twelve unpigmented) enabled assignment of paternity on this character alone. 


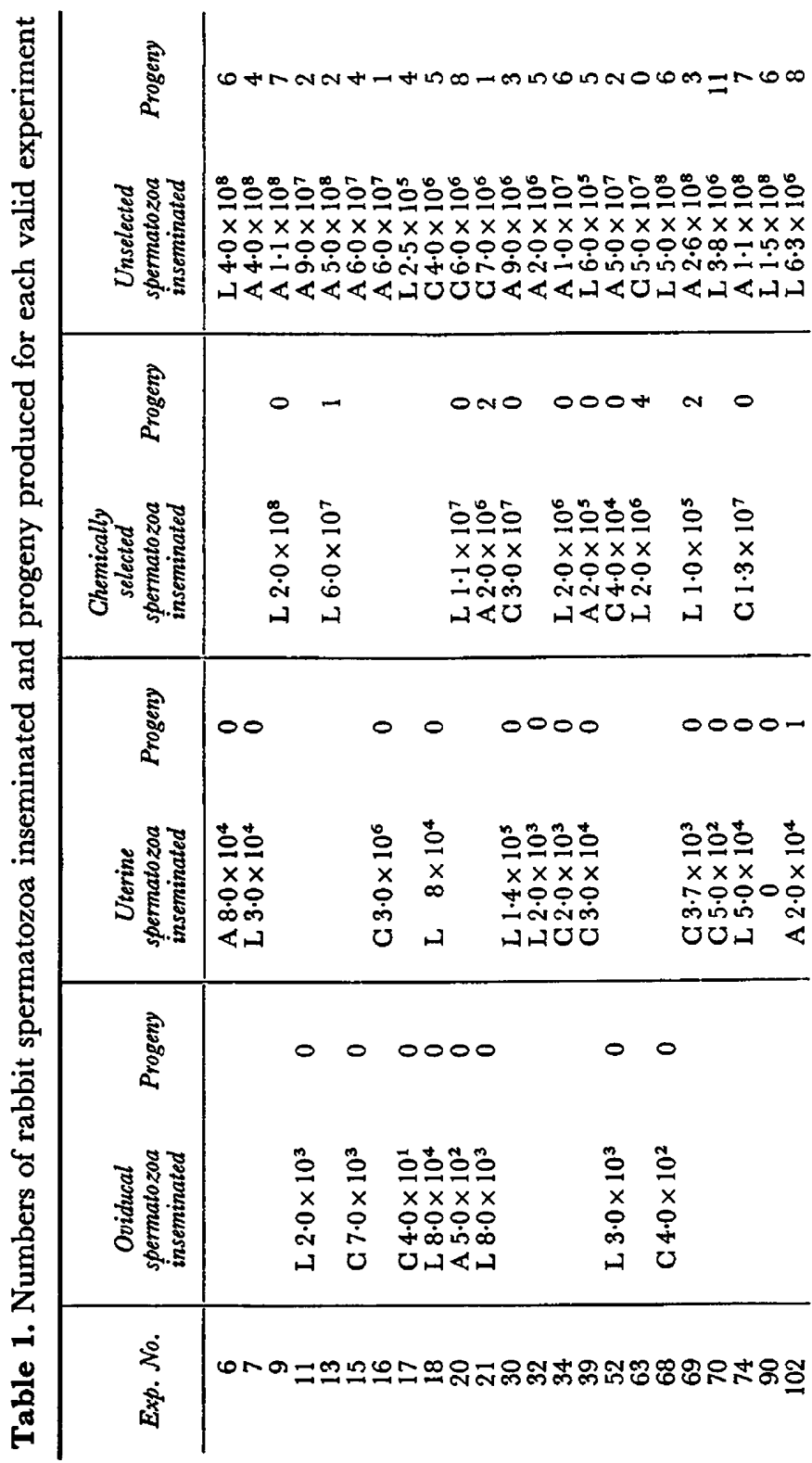




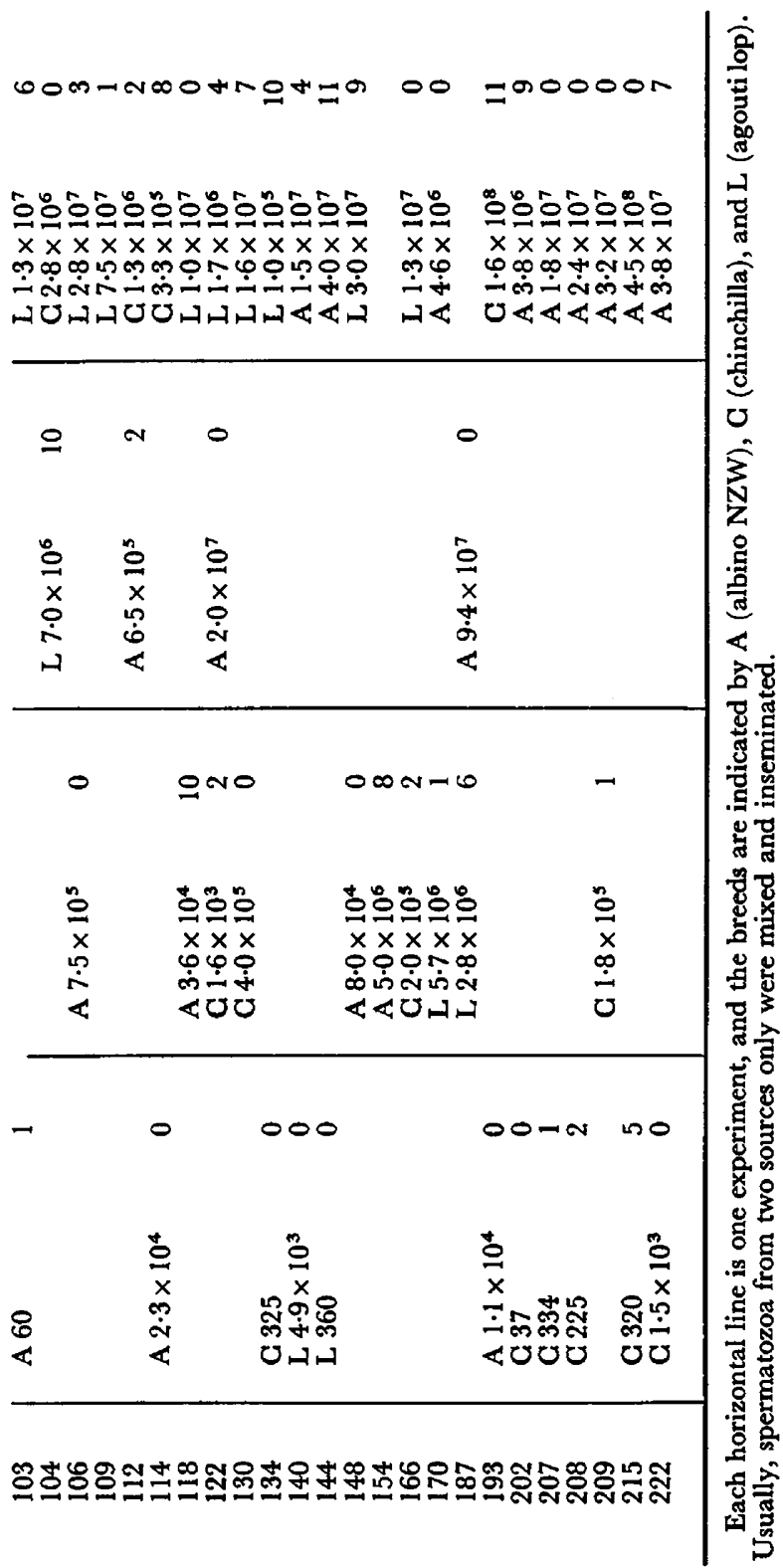




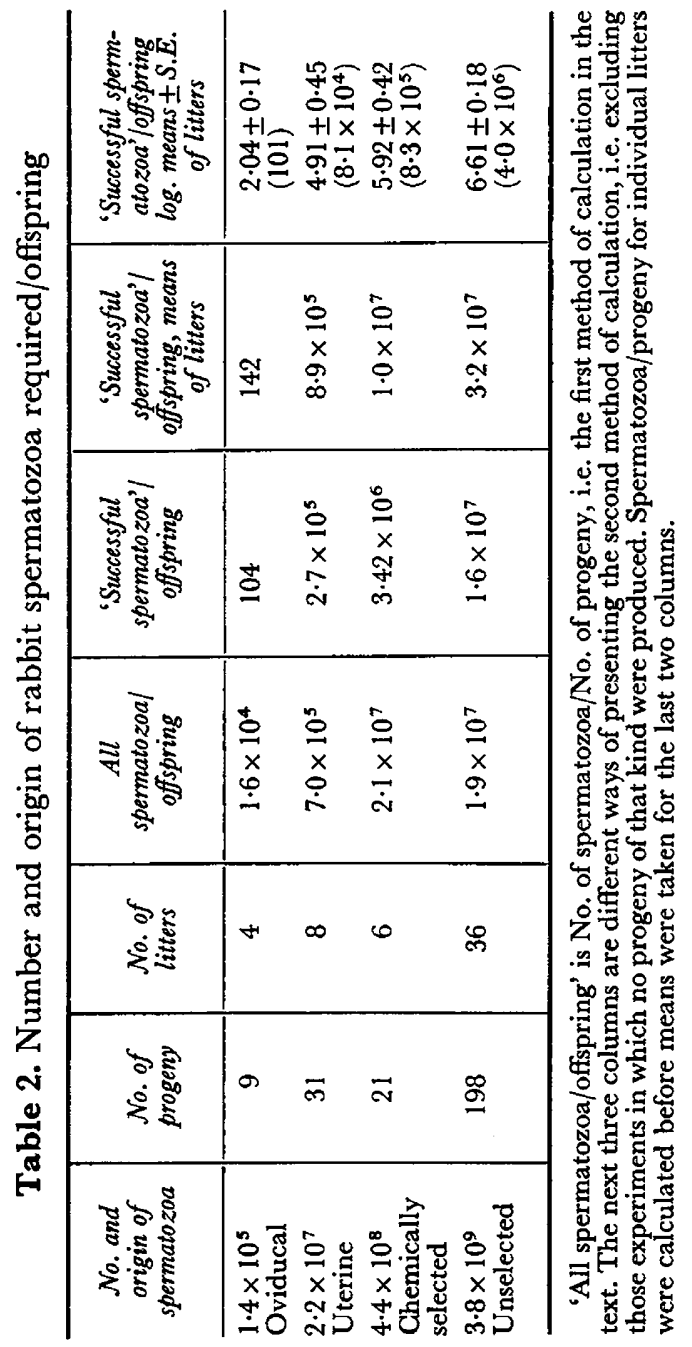


The progeny produced from spermatozoa recovered from the oviducts were also investigated for their immuno-alleles; these confirmed the paternity suggested by the ears and pigment in all cases.

The first litter containing such progeny was of note as it was a mixed litter: 60 NZW spermatozoa recovered from the oviducts mixed with $12.5 \times 10^{6}$ agouti lop ejaculated spermatozoa gave one and six offspring, judged by ear and pigmentation characters (Plate 1). The agouti lop had never produced white offspring in test litters while his heterozygous brother produced white offspring with lop ears as expected (Plate 1). Immuno-alleles were used as a further test on cryptic genetic characters. The NZW buck was homozygous at both loci, 1,1/4,4; the agouti-lop buck was $1,3 / 4,5$. The NZW doe was $1,3 / 4,4$. The white short-eared offspring was $1,1 / 4,4$. The other young were $1,1 / 4,4$; $1,1 / 4,4 ; 1,3 / 4,4 ; 1,1 / 4,5 ; 1,3 / 4,5 ; 1,3 / 4,5$.

\section{DISCUSSION}

Our experimental system worked in that all sperm samples were represented by litters, and there was no difficulty in establishing the paternity of the offspring. It was disappointing that the immuno-allele test did not prove as critical for paternity as colour and ear character (see Plate 1). Had the agouti lop been the father of the white offspring doe, the test had a $4: 1$ chance of disproving NZW paternity, but the doe's immuno-alleles were the same as those of two half-brothers. Nevertheless, the result favoured NZW paternity, by $4: 1$ to $16: 1$ depending on statistical assumptions, so this test proved less critical than the routine ones.

Only about a quarter of the does kindled, and since more than enough spermatozoa were always inseminated, we know that recipient females who produced no progeny were failures in experimental techniques. We will discuss, therefore, only those experiments which resulted in progeny. There was a notable lack of 'unselected' offspring accompanying the few 'selected' offspring in some cases (Exp. 215); ample unselected spermatozoa were inseminated.

Three factors could account for the high numbers of 'unselected' spermatozoa apparently required per offspring produced. Firstly, Overstreet (1970) found that the same numbers of spermatozoa inseminated in reduced volumes (to $0.01 \mathrm{ml}$ ) gave better results, but he inseminated by way of the fimbria; we usually inseminated about $1 \mathrm{ml}$ directly into the uterus. Secondly, Adams (e.g. 1969) has pointed out that too many spermatozoa around eggs may not only cause polyspermy, but even dissolution of the zona. The numbers of spermatozoa inseminated into the uterus in our experiments were usually very high, so there must always have been excess spermatozoa for the number of eggs available (cf. Wales et al., 1965) and many of the spermatozoa which were counted were effectively denied a chance of fertilizing. Therefore our figures may underestimate their effectiveness, but we always used less than an ejaculate.

There was a lack of mixed litters, only six of forty-seven. In most cases, there was presumably an effective preponderance of one sperm type, usually the 'unselected' type. This preponderance may not, of course, be reflected in actual sperm numbers; one or other sperm sample in our inseminate may have been 
killed or reduced in viability by our treatments, or may have contained fewer motile spermatozoa. Some samples may have come from a 'better' stud buck (Napier, 1961) but, because the bucks were used in rotation, this cannot account for the preponderance. More 'unselected' than 'selected' spermatozoa produced young, because their number approached that of a normal ejaculate; it was with this sample that we produced our required excess in numbers in nearly all the experiments.

These reasons for our low yield of progeny are well-founded, even on the null hypothesis that 'all spermatozoa are equal'. Our fewer selected spermatozoa, which had already sojourned in and been washed from one female tract, suffered in the same ways as our unselected spermatozoa, so their relative success is the more remarkable.

Two figures are available for sperm 'efficiency' in our system. The first includes counts of all inseminated spermatozoa of a given type and is divided by number of offspring, without any compensation for those cases where there were not enough eggs for these spermatozoa. Comparison of the figures in this way gives an extremely harsh view of the results, with a bias toward the 'unselected' spermatozoa. The figures are: 'unselected' $1.9 \times 10^{7}$, 'chemically selected' $2 \cdot 1 \times 10^{7}$, uterine $5.3 \times 10^{5}$, and oviducal $1.6 \times 10^{4}$. No standard error can be given because these are simple quotients.

Alternatively, the number of spermatozoa required to produce each of the offspring can be calculated. This produces a minimal, but comparable, estimate of the spermatozoa required per offspring, but without any allowance for those cases where no offspring were expected, either because the sperm sample was inadequate in numbers or because of statistical fluctuation. The bias introduced here is in the opposite direction. The figures are obtained by

\section{the number of spermatozoa in successful inseminates}

the number of progeny of that kind

and are: 'unselected' $1.6 \times 10^{7}$; 'chemically selected' $3.4 \times 10^{6}$; uterine $2.7 \times 10^{5}$; oviducal $1.04 \times 10^{2}$. These figures do not allow statistical comparisons to be made, so the litters have been analysed individually as

$$
\frac{\text { spermatozoa of that kind }}{\text { progeny of that kind }} \text {. }
$$

The logarithmic means and standard errors of all such figures for individual litters are given: 'unselected', $6 \cdot 61 \pm 0 \cdot 18$; 'chemically selected', $5 \cdot 92 \pm 0 \cdot 42$; uterine, $4 \cdot 97 \pm 0 \cdot 45$; oviducal $2 \cdot 04 \pm 0 \cdot 17$.

It is clear (significant at $1 \%$ level) that oviducal spermatozoa are better at fertilizing, in our system, than are uterine spermatozoa; these in turn are better (significant to $5 \%$ ) than 'chemically selected', spermatozoa which may not be distinguishable from 'unselected' spermatozoa. The use of logarithmic means, and of logarithms generally, is dictated by the nature of the numbers of spermatozoa obtained (Morton, 1970).

Unless 'super-capacitation' leading to 'super-fertility' is possible, of which there has been no hint in the literature (e.g. Bedford, 1970), we have shown that as spermatozoa are destroyed in the female tract, fertility is not lost proportion- 
ately and therefore that a small number of 'good' spermatozoa must be concentrated by the tract. The 'good' spermatozoa could be the precisely mature gametes, all others being juvenile or senile, or they could be a population which has succeeded in spermatogenesis, all others having failed in some way which makes them vulnerable in the female tract.

The first suggestion cannot be dismissed entirely. There are many results (Orgebin-Crist, 1969; Bedford, 1970) demonstrating the requirement for maturation of spermatozoa in the male tract and it is probable that many ejaculated spermatozoa are not properly mature. The requirement for physiological capacitation in the female tract is also agreed, but many spermatozoa seem to undergo their acrosomal changes while still very far from the egg (Bedford, 1970); such premature capacitation might render them incapable of fertilizing. Equally, many spermatozoa must delay the terminal stages of capacitation until too late. Consideration of the literature on storage, for example in the female bat (Racey, 1969) and male guppy (Billard, 1969), shows that months not minutes are involved for some species. From this, we might allow that a large proportion of the ejaculated spermatozoa are not useful gametes because of timing difficulties, but the main contribution to sperm heterogeneity must come from another source.

The viewpoint that small numbers of spermatozoa are released from a large store would assume that the trickling spermatozoa are representative of those in the store. Our experiments have shown, however, that spermatozoa high in the tract are a more fertile population, so that whilst such a trickle may be a part, it cannot be the whole of the reproductive strategy.

There is strong but circumstantial evidence that the extent of sperm wastage in the female tract is dependent upon the number of chiasmata in the production of each spermatozoon (Cohen, Jack, 1967, 1969, 1973). If this were so, sperm selection by the female rabbit would be expected. Even though the agreement between the chiasma/sperm wastage theory and our rabbit results is encouraging, we are puzzled in three areas.

Firstly, the rabbit female genital tract contrives to prevent most spermatozoa from reaching the fertilization site. We do not understand the mechanism, but can view the successive barriers of cervix, uterus, and uterotubal junction as selective. In this, we differ from Johnson \& Hunter (1972) who found antigenic differences in the reduced sperm populations high in the tract and concluded that each spermatozoon changed antigenically.

Secondly, we are puzzled by the situation in species such as the mouse or bull in which the semen is deposited directly into the uterus or cervix and only one barrier seems to be presented to the spermatozoa. D. J. Werrett (unpublished observations) is now finding that, in the mouse, sperm destruction occurs mostly after fertilization.

The third puzzling area is that of 'external fertilization'. Presumably, some species using this method select spermatozoa in their passage through such egg coats as the albumin in the frog, or by agglutination as in the sea urchin (Cohen, Jack, 1969).

These are clearly puzzling problems for any theory of sperm number; we believe that future explanations must be based on selection of spermatozoa by the female. 


\section{ACKNOWLEDGMENTS}

The Schering Corporation supported the initiation of this work, and the Medical Research Council has since continued this support (grant no. 0969/ 148/B).

We wish to express our indebtedness to them, and to Mrs Judith Dawson, Dr J. Marston and Dr D. Catty who helped to develop the successful technique.

Dr R. V. Short and Dr R. A. Beatty commented very helpfully on the first draft of this paper, and we have gratefully adopted most of their suggestions.

\section{REFERENCES}

AdAms, C. E. (1969) Intraperitoneal insemination in the rabbit. F. Reprod. Fert. 18, 333.

Adams, G. E. (1972) The rabbit. In The U.F.A.W. Handbook on the Care and Management of Laboratory Animals, pp. 167-186. Ghurchill-Livingstone, Edinburgh.

Ausrin, C. R. (1965) Fertilisation. Foundations of Developmental Biology Series. Prentice-Hall, New Jersey.

BedFord, J. M. (1965) Effect of environment on phagocytosis of rabbit spermatozoa. F. Reprod. Fert. 9, 249.

BeDford, J. M. (1970) Sperm capacitation and fertilisation in mammals. Biol. Reprod., Suppl. 2, 128.

Billard, R. (1969) La spermatogènese de Poecilia reticulata. Annls Biol. anim. Bioch. Biophys. 9, 307.

Braden, A. W. H. \& Austin, G. R. (1954) Number of sperms about the eggs in mammals and its significance for normal fertilisation. Aust. F. biol. Sci. 7, 543.

Casaret, G. W. (1953) A one-solution stain for spermatozoa. Stain Technol. 28, 125.

CoHen, JACK (1967) Correlation between sperm 'redundancy' and chiasma frequency. Nature, Lond. $215,862$.

Cohen, JACK (1969) Why so many sperms? An essay on the arithmetic of reproduction. Sci. Prog. 57, 23.

CoHEN, JACK (1971) The comparative physiology of gamete populations. Adv. comp. Physiol. Biochem. 4, 267.

Cohen, JACK (1973) Crossovers, sperm redundancy, and their close association. Heredity, Lond. 31, 408.

Cohen, Jean (1969) Immunological factors and unexplained sterility. Acta europ. fertil. 1, 193.

Edwards, R. G. (1967) Antibodies and fertility. Sci. $\mathcal{F l}, \mathbf{3}, 69$.

Edwards, R. G., Bavister, B. D. \& Steptoe, R. G. (1969) Early stages of fertilization in vitro of human oocytes matured in vitro. Nature, Lond. 221, 632.

HARPER, M.J. K. (1963) Ovulation in the rabbit: the time of follicular rupture and expulsion of eggs, in relation to injection of luteinizing hormone. F. Endocr. 26, 307.

Johnson, W. L. \& Hunter, A. G. (1972) Seminal antigens: their alteration in the genital tract of female rabbits and during partial in vitro capacitation with beta amylase and beta glucuronidase. Biol. Reprod. 7, 332.

Mann, T. (1964) The Biochemistry of Semen and of the Male Reproductive Tract. Methuen, London.

Morton, D. B. (1970) On the transport of spermatozoa in the female rabbit. Ph.D. thesis, University of Liverpool.

NAPIER, R. A. N. (1961) Fertility in the male rabbit. III. Estimation of the spermatozoan quality by mixed insemination and the inheritance of spermatozoan character. F. Reprod. Fert. 2, 273.

Orgebin-Grist, M.-G. (1969) Studies on the function of the epididymis. Biol. Reprod., Suppl. 1, 155.

Overstreet, J. W. (1970) Sperm numbers and fertilization in the rabbit. F. Reprod. Fert. $21,279$.

PaRker, G. A. (1970) Sperm competition and its evolutionary consequences in the insects. Biol. Rev. 45, 525.

RACEx, P. A. (1969) Diagnosis of pregnancy and the experimental extension of gestation in the pipistrelle bat, Pipistrellus pipistrellus. F. Reprod. Fert. 19, 465.

Restall, B. J. (1967) The biochemical and physiological relationships between the gametes and the female reproductive tract. Adv. reprod. Physiol. 2, 181.

Soupart, P. (1967) Studies on the hormonal control of rabbit sperm capacitation. $\mathcal{F}$. Reprod. Fert., Suppl. 2, 49.

Symons, D. B. A. (1967) Reactions of spermatozoa with uterine and serum globulin determined by immunofluorescence. F. Reprod. Fert. 14, 163.

Wales, R. G., Martin, L. \& O'SheA, T. (1965) Effect of dilution rate and of the number of spermatozoa inseminated on the fertility of rabbits ovulated with chorionic gonadotrophin. $\mathcal{F}$. Reprod. Fert. 10, 69 . 